\title{
Isolated Tear of the Distal Biceps Femoris Tendon in a Young Elite Australian Rules Football Player
}

\author{
In Sun $\mathrm{Na}^{1,2^{*}}$, Stuart Down ${ }^{3}$ and Scott Hancock ${ }^{2,4}$ \\ ${ }^{1}$ Department of Rehabilitation Medicine, Alfred Health, Australia \\ ${ }^{2}$ Department of Sport Medicine, Oakleigh Chargers Football Club, Australia \\ ${ }^{3}$ Department of Sport and Exercise Medicine, Lifecare Ashburton Sports Medicine, Australia \\ ${ }^{4}$ Department of Physiotherapy, Kieser Mont Albert, Australia
}

*Corresponding author: In Sun Na, Department of Rehabilitation Medicine and Sport Medicine, Alfred Health, Oakleigh Chargers Football Club, 506/83-85 Drummond Street, Oakleigh, VIC, Australia 3166, Tel: +61-403456744

\begin{abstract}
Isolated tear of the distal biceps femoris tendon is rare in athletes. A recent systematic review identified only a total of 22 athletes with such injuries. More specifically, only one previously reported case in an athlete outlined a tear localised to the distal tendon, as opposed to the more commonly documented myotendinous junction. Most of these injuries were treated surgically $(n=20 / 22)$ with mean $( \pm$ SD) overall time to return to sport of $4.9+/-3.3$ months. This study presents a rare case of an isolated tear of the distal biceps femoris tendon in an elite athlete under the age of 18 . It also presents a case of successful non-surgical management of an isolated tear localised to the distal biceps femoris tendon. Time to return to pre-injury level of sport was only 35 day (approximately 1 month). This case report invites consideration that not all isolated tears of the distal biceps femoris tendon in athletes necessitate surgical management, especially partial-width injuries localised within the distal tendon. Under certain clinical circumstances, non-operative management may provide equally successful clinical outcomes and relatively shorter timeframes for return to sport; a far more palatable option for the athlete.
\end{abstract}

\section{Introduction}

The hamstring muscle complex consists of the biceps femoris, the semitendinosus and the semimembranosus [1]. It serves as a hip extensor, knee flexor and external rotator of the hip and knee depending on the leg positioning [2]. It is one of the most frequently injured muscles in athletes, accounting for significant periods of absence from competition [3-6]. This is particularly true for athletes involved in running, jumping, kicking and rapid acceleration [3]. More cases of proximal hamstring injuries are reported in the literature, whilst injuries to the distal hamstring are limited to small case series and case reports [3,4,7-9].

Isolated injuries of the distal biceps femoris tendon are particularly rare in athletes [3]. Injuries may range from simple strains to partial or complete tearing of the musculotendinous junction or tendon avulsions from the head of the fibula $[3,10]$. Knapik, et al. conducted a systematic review and identified a total of 22 athletes with isolated distal biceps femoris tendon tearing sustained during sporting activities from December 1970 to December 2017 [3]. More specifically, only one previously reported case in an athlete outlined a tear localised to the distal tendon, as opposed to the more commonly documented myotendinous junction [3]. The vast majority $(n=20 / 22)$ of reported cases were treated surgically with mean $( \pm S D)$ overall time to return to sport of $4.9+/-3.3$ months [3].

In our case report, we discuss a rare case of an isolated tear of the distal biceps femoris tendon in a young elite Australian Rules Football playerunder the age of 18 , and its successful non-surgical management.

\section{Case Description}

A 16-year-old right foot dominant male Australian Rules Football player experienced pain tothe lateral as-

Citation: Na IS, Down S, Hancock S (2021) Isolated Tear of the Distal Biceps Femoris Tendon in a Young Elite Australian Rules Football Player. Int J Sports Exerc Med 7:182. doi.org/10.23937/24695718/1510182

Accepted: January 14, 2021; Published: January 16, 2021

Copyright: (C) $2021 \mathrm{Na}$ IS, et al. This is an open-access article distributed under the terms of the Creative Commons Attribution License, which permits unrestricted use, distribution, and reproduction in any medium, provided the original author and source are credited. 
pect of his right knee over a one week period. The subject was a high school student playing Australian Rules Football at an elite competitive level for one of the Under-18 (U18) Australian Football League (AFL) clubs in addition to another local club. He did not recall a specific traumatic event heralding the onset of his symptoms, but continued to report activity-related lateral pain to his right knee, more specifically in the region of the head of the fibula. In the aftermath of his last game prior to specialist assessment, the subject had struggled to run, jump, change direction, squat and also to sit in a chair for several days. He also noted mild localised swelling around the fibular head region. He did not report any mechanical or instability symptoms, denied any previous musculoskeletal injuries and was generally in excellent health.

On examination, the head of the fibulawas slightly more protuberant on the right side, and this was more pronounced in mid-squat position. Whilst the superior tibiofibular joint was not unstable to ballottement, there was moderate tenderness to the posterior fibular head and distal biceps femoris tendon. There was no effusion or bruising in the area. There was no specific joint line tenderness or rotatory discomfort, and all major ligaments were grossly intact with firm end point. The posterolateral corner was stable with a negative dial test, and there was no significant increased laxity with tibial rotation. Meniscal tests were negative. Pain localising to the fibular head was provoked on resisted prone knee flexion, particularly nearing terminal extension.

Likely differential diagnoses for lateral knee pain in athletes include lateral meniscal injury, injuries to posterolateral corner structures (e.g. popliteus, lateral collateral ligament, mid third capsular ligament (anterolateral ligament), iliotibial band syndrome and biceps femoris tendinopathies [11]. Based on our clinical findings, an injury to his distal biceps femoris tendon was suspected. Magnetic Resonance Imaging (MRI) of his symptomatic knee was organised to further clarify and characterise his injury, as both the management plan and prognosis for an in-season elite athlete can vary significantly according to the pathology and its severity.

\section{Investigation}

As suspected based on the clinical findings, MRI confirmed pathology involving the biceps femoris tendon, albeit a full thickness partial width tear of the posterior fibular attachment involving approximately $30 \%$ of the tendon width. This was associated with a bleed extending superior to the musculo tendinous junction (Figure 1 and Figure 2).

$\mathrm{MRI}$ also revealed marrow oedema to the posteromedial tibial plateau beneath a healthy-looking meniscus (Figure 3A). Although high signal changes were present within the posterior horn of the medial meniscus, these did not reach either articular surface to satisfy

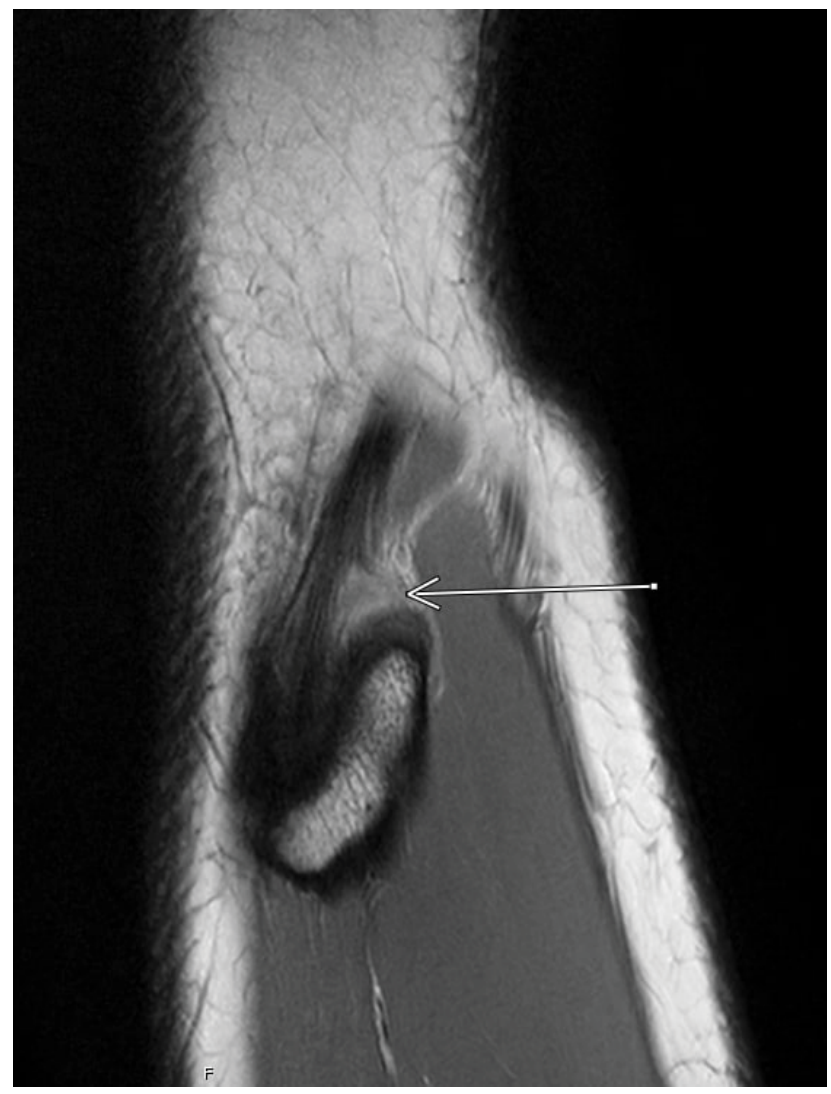

Figure 1: Sagittal PD-weighted SPAIR (SPectral Adiabatic Inversion Recovery) image of partial width tear of the posterior biceps femoris fibular attachment.

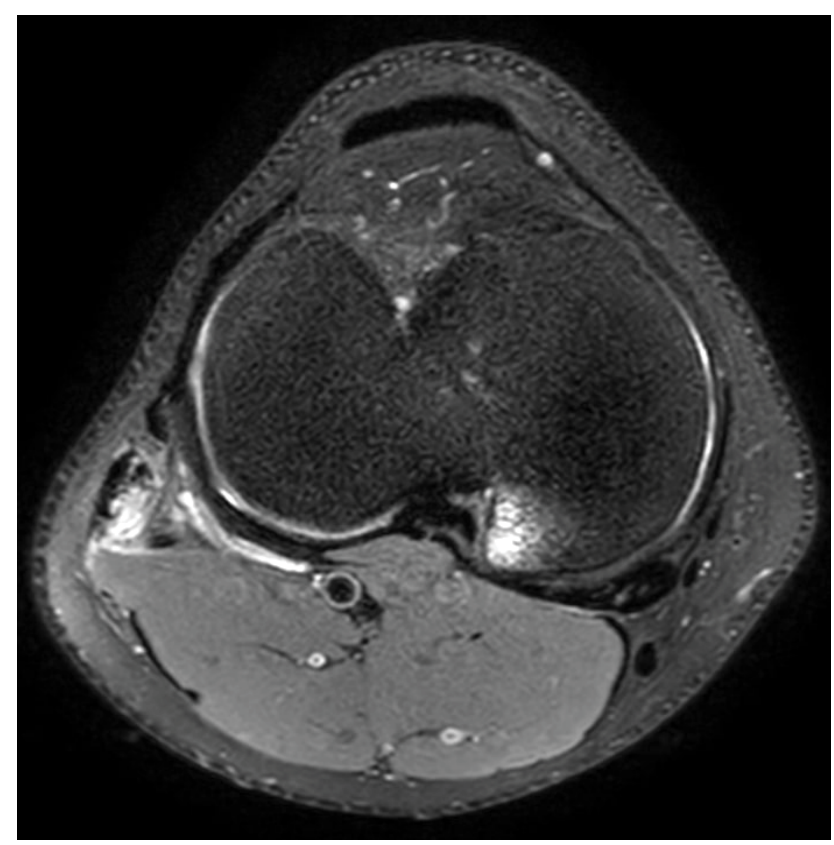

Figure 2: Axial PD-weighted SPAIR image of partial width tear of the posterior biceps femoris fibular attachment and bone bruising around posteromedial tibial plateau area.

an MRI diagnosis of meniscal tear (Figure 3B). Furthermore, clinical exams tested negative for meniscal injury, and therefore this signal was felt to be in keeping with prominent vasculature, commonly seen in this young age group [12]. 


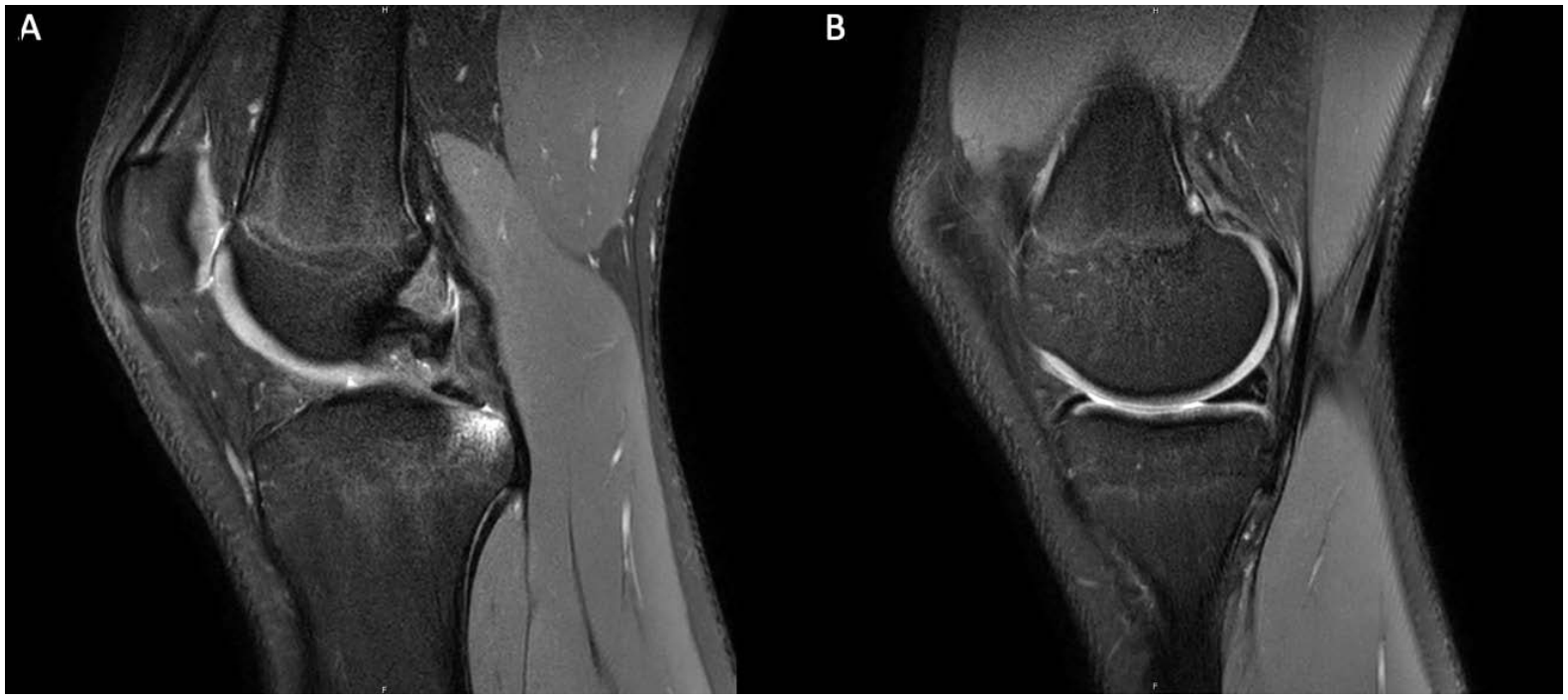

Figure 3: Sagittal PD-weighted SPAIR image of (A) Bone bruising around posteromedial tibial plateau area; (B) Signal changes within the posterior horn of the medial meniscus.

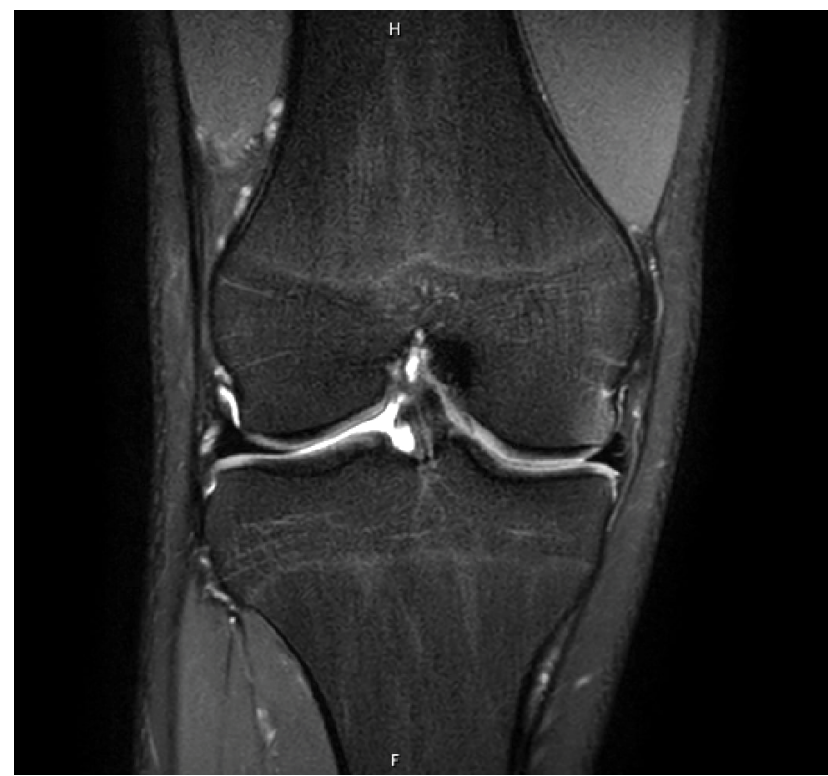

Figure 4: Coronal PD-weighted SPAIR image of marginal marrow oedema to the inferomedial femoral condyle and adjacent medial meniscocapsular junction.

In addition, we found marginal marrow oedema to the inferomedial femoral condyle and adjacent medial menisco-capsular junction on MRI (Figure 4). We hypothesise this may have been from a direct contusion (in turn, possibly resulting in varus stress to the knee and damage to biceps femoris tendon) or possibly as part of a hyperextension and twist type mechanism of injury. As mentioned earlier, however, our subject could not explicitly recall a knee injury at any stage, rather progressively worsening knee pain and function.

We also noted low level hyperintensity change through the Anterior Cruciate Ligament (ACL), which may reflect some local loculation of joint fluid in the notch or possibly a very low grade strain of the ACL (Figure 5).

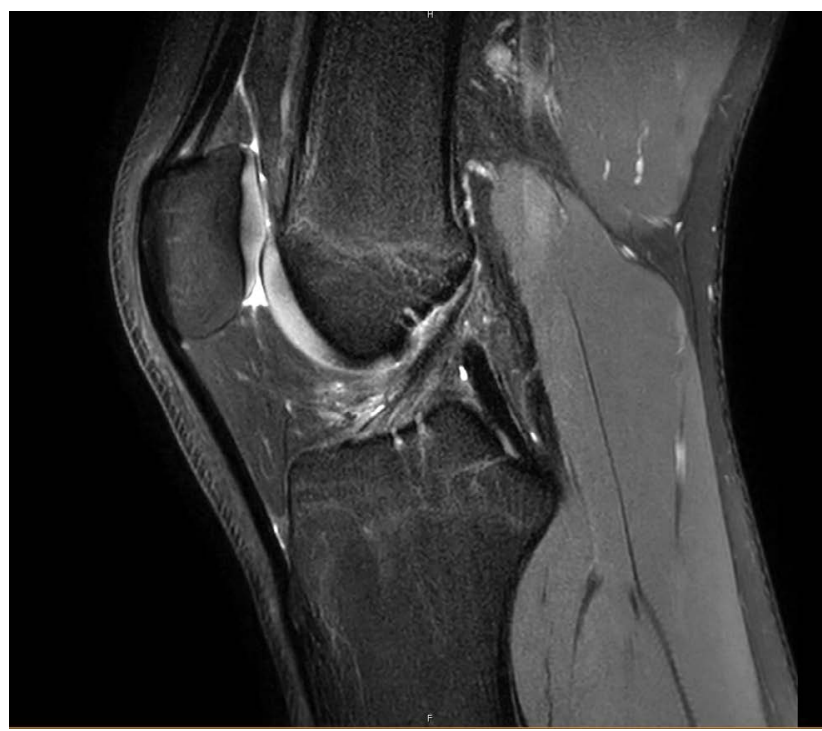

Figure 5: Sagittal PD-weighted SPAIR image of low level hyperintensity change through the anterior cruciate ligament.

\section{Outcome}

Given the apparent rarity of this specific injury, there is no established or well-documented algorithm for the management of isolated tears of the distal biceps femoris tendon. Hence, with assessment and advice from an experienced Sport and Exercise Medicine Physician, we formulated an individualised rehabilitation program. Rather than setting rehabilitation goals based on objective measures (e.g. isokinetic testing, strength testing), we primarily focused on graded return to sport based on activity-based goals.

Initial phase of rehabilitation primarily focused on two weeks of off-loading the injured knee from lower limb impact activities and forceful hamstring contraction to achieve participation in daily activities without pain. During this period, the subject abstained from jogging, 
running and ballistic activities but continued to actively work on general fitness and leg strength by means of un-cleated in-saddle cycling, swimming and cross-training. The subject also continued to participate in gym activities, mainly upper body, with avoidance of weighted lunges, weighted squats and weighted dead lifts.

After the two weeks of off-loading period, the subject was performing daily activities (e.g. walking/use of stairs/sitting) without any pain or discomfort. There was still trivial tenderness to firm palpation of the distal biceps femoris tendon and fibular head at this point. Otherwise, he was able to perform full passive and active range of motion on the injured knee without any pain. Additionally, he was able to perform ballistic-style kicking, heavy resisted prone knee flexion with and without lateral compartment bias and varus stress testing without provoking pain. The subject successfully participated in a half session of non-contact training on the same day, starting with graduated return to pain-free running from jogging.

Two days after his participation in modified training, the subject then successfully completed a full training session including the usual contact components. Subsequently, three days after his full training, the subject successfully returned to playing at a local club level for just over half a game without any exacerbation of symptoms. The subject's injured knee was taped to offload varus force for all training sessions and game play.
Overall, the subject gradually returned to progressive pain-free running and full trainingover a week after an initial off-loading period of two weeks, and returned to play football at club level 29 days after his reported 'injury' (being the last game of football in which he played prior to reporting his injury to team medical staff).

Time to return to his pre-injury level of sport was 35 days, on the basis that he was required to play at least half a game at lower club level before returning to his usual elite U-18 level. For the remaining 3 months of the season, the subject did not sustain re-injury to his distal biceps femoris tendon, or for that matter report further issues with his knee. At the time of writing this report, our subject has remained symptom-free with respect to this knee and continued to train and play in the same elite U-18 team until nation-wide restrictions were set in place to stop all team sport training and participation due to the COVID-19 pandemic situation.

It is interesting and noteworthy that our subject did not report a specific injury per se, rather a worsening localised lateral knee pain and functional difficulty. Given our subject could not recall a specific injury, his presentation may have been attritional weakening of the tendon over time, though his young age and the other accompanying MRI changes suggest this was far more likely an acute injury that seemingly escaped his attention within the rough and heavy-contact environment that is AFL football. Whilst we appreciate there may

Table 1: Graded return to sport based on activity-based goals by days after the injury.

\begin{tabular}{|c|c|c|}
\hline Day(s) & Signs/Activity level & Rehabilitation Principles \\
\hline $0-10$ & $\begin{array}{l}\text { - Daily activities limited by lateral knee pain (struggled to sit, } \\
\text { squat, run, jump or change direction). }\end{array}$ & $\begin{array}{l}\text { - Did not seek medical attention. } \\
\text { - Self-initiated stopping painful activities. }\end{array}$ \\
\hline 10 & $\begin{array}{l}\text { - Mild improvement but ongoing activity-related lateral knee } \\
\text { pain. } \\
\text { - Moderate tenderness to the posterior fibular head and } \\
\text { distal biceps femoris tendon. } \\
\text { - Pain provoked on resisted prone knee flexion, particularly } \\
\text { nearing terminal extension. }\end{array}$ & $\begin{array}{l}\text { - } 2 \text { weeks of off-loading the injured knee from impact } \\
\text { and forceful hamstring contraction. } \\
\text { - Abstained from jogging, running and ballistic activities. } \\
\text { - Continued to work on general fitness and leg strength } \\
\text { by means of un-cleated cycling, swimming and cross } \\
\text { training. } \\
\text { - Continued to participate in gym activities, mainly } \\
\text { upper body, with avoidance of weighted lunges, } \\
\text { weighted squats and weighted dead lifts. }\end{array}$ \\
\hline 24 & $\begin{array}{l}\text { - Performing daily activities (e.g. walking) without any pain } \\
\text { or discomfort. } \\
\text { - Trivial tenderness to firm palpation of the distal biceps } \\
\text { femoris tendon and fibular head. } \\
\text { - Able to perform full passive and active range of motion on } \\
\text { the injured knee without any pain. } \\
\text { - Able to perform ballistic-style kicking, heavy resisted prone } \\
\text { knee flexion with and without lateral compartment bias and } \\
\text { varus stress testing without provoking pain. } \\
\text { - Participated in half training, starting with graduated return } \\
\text { to pain-free running from jogging. }\end{array}$ & $\begin{array}{l}\text { - As above, except allowed to jog and run as long as } \\
\text { pain free. } \\
\text { - The subject's injured knee was taped to offload varus } \\
\text { force for all training sessions. }\end{array}$ \\
\hline 26 & - Participated in full training including the contact component. & - Full training as long as pain free. \\
\hline 29 & $\begin{array}{l}\text { - Returned to playing AFLat a local club level for at least a } \\
\text { half a game. }\end{array}$ & - Full training as long as pain free. \\
\hline
\end{tabular}


have been an element of good fortune in our subject's rapid return to sport, he carried out appropriate, guided rehabilitation with progressive loading and passed all clinical tests prior to a return to both training and game play (Table 1).

\section{Discussion}

As discussed earlier, isolated tears of the distal biceps femoris tendon is rare in athletes [3]. More specifically, almost all of the previously reported cases of isolated distal biceps femoris injuries record tearing at the musculocutaneous junction or complete tendon avulsions from the fibular head [3]. This can be explained by the fact that the forces are more likely to cause an avulsion fracture or tearing at the musculotendinous junction as the tensile strength of the tendon is so great that an acute tear of a normal, healthy tendon is rare [11]. It is believed that the tendons that do acutely tear usually have some form of underlying pathology [11]. Therefore, tearing of the tendons most frequently occur in middle-aged and older patients [11].

Interestingly, our otherwise healthy 16-year-old athlete sustained a partial tearing injury localised within the distal biceps femoris tendon. To date, only one previously reported case outlined a tear localised within the distal tendon in an athlete: A 24-year-old male soccer player with partial tearing $[3,13]$. This previously reported case was treated surgically, requiring a 5-month rehabilitation period to return to sport $[3,13]$.

This case is novel in that it reports a successful case of non-surgical management of an isolated partial tear localised within the distal biceps femoris tendon in an elite athlete. Compared to the previously reported case $[3,13]$, where the injury was managed operatively, the time to return to sport was significantly shorter for our subject, by nearly 4 months. It is also worth noting that the vast majority ( $n=20 / 22$ ) of reported cases of isolated tearing of the distal biceps femoris tendon in athletes were treated surgically with mean $( \pm S D)$ overall time to return to sport of $4.9+/-3.3$ months [3], far longer than was documented with our subject ( 35 days).

\section{Conclusion}

This case report invites consideration that not all isolated tears of the distal biceps femoris tendon in athletes necessitate surgical management, especially partial-width injuries localised within the distal tendon. It will also remind clinicians that every case requires an individualised management plan based on specific objective and subjective findings coupled with radiological information to aid decision making. Under certain clinical circumstances and with appropriate guidance from experienced Specialists, non-operative management may provide equally successful clinical outcomes and relatively shorter timeframes for return to sport; a far more palatable option for the athlete.

\section{Acknowledgement}

The primary author would like to acknowledge the medical staff at OCFC (Sue Allan and Declan Callahan) for their care and dedication looking after the players in the club, including the subject.

The primary author would also like to acknowledge the coaching staff and managers at OCFC (Leigh Clarke, Jy Bond, James Honey, Alan Morris and Alan Johnson) for their support to the medical team and the players.

\section{Contributorship Statement}

ISN is the first author of this article.

SD is the second author and supervisor of this article.

$\mathrm{SH}$ is the third author of this article.

All authors contributed to conception, drafting, revising and finalising the article.

All authors were also involved in patient care and injury management.

\section{References}

1. Ernlund L, Vieira LA (2017) Hamstring injuries: update article. Rev Bras Ortop 52: 373-382.

2. Malliaropoulos NG (2013) Non contact Hamstring injuries in sports. Muscles Ligaments Tendons J 2: 309-311.

3. Knapik DM, Metcalf KB, Voos JE (2018) Isolated Tearing and Avulsion of the Distal Biceps Femoris Tendon During Sporting Activities: A Systematic Review. Orthop J Sports Med 6: 2325967118781828.

4. Cohen S, Bradley J (2007) Acute proximal hamstring rupture. J Am Acad Orthop Surg 15: 350-355.

5. Volpi P, Melegati G, Tornese D, Bandi M (2004) Muscle strains in soccer: a five-year survey of an Italian major league team. Knee Surg Sports Traumatol Arthrosc 12: 482-485.

6. Askling C, Karlsson J, Thorstensson A (2003) Hamstringinjuryoccurrencein elite soccer players after preseason strength training with eccentric overload. Scand J Med Sci Sports 13: 244-250.

7. Alzahrani MM, Aldebeyan S, Abduljabbar F, Martineau PA (2015) Hamstring injuries in athletes: Diagnosis and Treatment. JBJS Rev 3.

8. Clanton TO, Coupe KJ (1998) Hamstring strains in athletes: diagnosis and treatment. J Am Acad Orthop Surg 6: 237-248.

9. De Smet AA, Best TM (2000) MR imaging of the distribution and location of acute hamstring injuries in athletes. AJR Am J Roentgenol 174: 393-399.

10. Lempainen L, Banke IJ, Johansson K, Brucker PU, Sarimo J, et al. (2015) Clinical principles in the management of hamstring injuries. Knee Surg Sports Traumatol Arthrosc 23: 2449-2456.

11. Brukner P, Khan K, Clarsen B, Cook J, Cools A, Crossley K, et al. (2017) Clinical Sports Medicine. ( $5^{\text {th }}$ edn), North Ryde, New South Wales: McGraw-Hill Australia.

12. Pai DR, Strouse PJ (2011) MRI of the Pediatric Knee. American Journal of Roentgenology 196: 1019-1027.

13. Lempainen L, Sarimo J, Mattila K, Heikkila J, Orava S, et al. (2007) Distal tears of the hamstring muscles: review of the literature and our results of surgical treatment. $\mathrm{Br} \mathrm{J}$ Sports Med 41: 80-83. 